\title{
Avaliação da presença da Síndrome de Burnout em Agentes Comunitários de Saúde no município de Aracaju, Sergipe, Brasil
}

\author{
Assessment of the prevalence of burnout syndrome in community \\ health agents of the city of Aracaju in the state of Sergipe, Brazil
}

Caroline Mascarenhas Mota ${ }^{1}$

Giselle Santana Dosea ${ }^{2}$

Paula Santos Nunes ${ }^{2}$

${ }^{1}$ Residência Integrada Multiprofissional em Saúde Coletiva, Universidade Tiradentes. Av. Murilo Dantas 300, Farolândia. 49032-490 Aracaju Sergipe Brasil.carol.mota@hotmail. com

${ }^{2}$ Faculdade de Fisioterapia, Universidade Tiradentes.
Abstract The Community Health Worker Program was established in 1991 with the aim of contributing to improving the quality of life of the population, with the community health workers being the program's protagonists. In line with this, this study aimed to evaluate the prevalence of burnout syndrome in the community health workers in Aracaju. Data collection was conducted among 222 community health workers in 43 family health units in Aracaju. The research instruments were: identification sheets produced by the authors of the research to verify the occupational and socioeconomic situation of the professionals; Job Stress Scale Questionnaires and the Maslach Burnout Inventory. The results showed that in relation to exposure to stress, $57.2 \%$ experienced psychological pressure and marked job control; $10.8 \%$ of community health workers showed a moderate tendency to Burnout Syndrome and 29.3\% manifested characteristics equivalent to the syndrome. These characteristics of burnout syndrome may be related to frustration with respect to inefficacy in resolving the problems and the close involvement of community health workers with their community. Given these facts, it is necessary to create intervention strategies that seek to prevent the psychosocial problems encountered in these health professionals.

Key words Occupational stress, Burnout syndrome, Community health workers
Resumo O Programa de Agentes Comunitários de Saúde nasceu em 1991 com o objetivo de contribuir para melhoria da qualidade de vida da população, tendo como protagonistas os agentes comunitários de saúde. Diante disso, este trabalho teve como objetivo avaliar a presença da Síndrome de Burnout em agentes comunitários de saúde de Aracaju (SE). A coleta de dados foi realizada com 222 agentes comunitários de saúde das 43 unidades de saúde da família de Aracaju. Os instrumentos da pesquisa foram: ficha de identificação produzida pelos autores para verificar a situação socioeconômica e ocupacional dos profissionais; questionários Job Stress Scale e Maslach Burnout Inventory. Os resultados mostraram que em relação à exposição ao estresse, 57,2\% apresentam alta demanda psicológica e alto controle sobre o trabalho; 10,8\% dos agentes comunitários de saúde demonstram moderada tendência à Síndrome de Burnout e 29,3\% apresentam características equivalentes à doença. Essas características podem estar relacionadas à frustação relacionada à ineficácia da resolutividade dos problemas e ao grande envolvimento dos agentes comunitários de saúde com a sua comunidade. Diante disso, é necessário criar estratégias de intervenção que busquem a prevenção de problemas psicossociais presentes nesses profissionais da saúde.

Palavras-chave Estresse ocupacional, Sindrome de Burnout, Agentes Comunitários de Saúde 


\section{Introdução}

O Programa de Agentes Comunitários de Saúde (PACS) nasceu em 1991, a partir de uma experiência que surgiu no Ceará, com o objetivo de contribuir para melhorar a qualidade de vida da população, investindo na educação em saúde, tendo como peça fundamental os agentes comu-

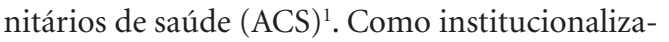
ção desse programa, em 1994, surge o Programa de Saúde da Família (PSF), atualmente chamado de Estratégia de Saúde da Família (ESF), com a finalidade de organizar a atenção básica no país e ratificar os princípios do SUS, garantindo, assim, o bem estar individual e coletivo, através de ações preventivas integrais e contínuas, tirando o foco do modelo biomédico vigente no país ${ }^{2}$.

A Portaria no 2.488, de 21 de outubro de 2011 estabelece a revisão de diretrizes e normas para a organização da Atenção Básica para a ESF e o PACS, assim como define as atribuições de cada categoria profissional existente na equipe de saúde da família. Os ACS têm entre suas funções: cadastrar todas as pessoas de sua microárea e manter os cadastros atualizados; desenvolver ações de integração entre a equipe de saúde e a população adscrita à Unidade Básica de Saúde; desenvolver atividades de promoção da saúde, de prevenção das doenças e de agravos e de vigilância à saúde, através de ações educativas individuais e coletivas na comunidade ${ }^{3}$.

Os ACS são o elo entre a equipe e a comunidade, fazendo a ligação entre o saber científico e o popular ${ }^{4}$. São os profissionais que estão em maior contato com a comunidade por residir na mesma área de atuação; são facilitadores do acesso aos serviços de saúde e, muitas vezes, os primeiros a ouvirem as queixas dos usuários na atenção primária ${ }^{2}$. A equipe e a comunidade depositam grandes anseios e expectativas nos ACS, os quais podem vir a se tornar fortes estressores para a saúde desses profissionais ${ }^{5}$.

Outras dificuldades encontradas por esse profissional são: quantidade elevada de pessoas da comunidade atendida, associada à falta de entendimento da população quanto ao seu trabalho; dificuldade de resolução dos problemas da comunidade, que depende do envolvimento de toda equipe multidisciplinar; falta de organização do serviço e relações conflituosas na equipe. Os ACS, também, compartilham o mesmo contexto social e cultural da população adscrita à unidade de saúde da família, por residirem no mesmo bairro de atuação ${ }^{1}$.
Além disso, na sociedade contemporânea prioriza-se a produtividade em detrimento da qualidade dos serviços e as atividades laborais ocupam um tempo considerável da vida do ser humano ${ }^{6}$. Esse conjunto de fatores relacionados ao trabalho provocam desgastes físicos e psicológicos, podendo ocasionar, posteriormente, transtornos mentais e comportamentais, como a Síndrome de Burnout ${ }^{7}$.

Os estudos relacionados à Síndrome de Burnout começaram a se destacar porque esclareceram os impactos da vida laboral na saúde do trabalhador, mostrando de que forma isso poderia interferir em seu desempenho no trabalho, seja na qualidade do serviço ou nos níveis de produção e, consequentemente, na saúde daquelas pessoas pelas quais eles são responsáveis ${ }^{6}$.

A Síndrome de Burnout, também chamada síndrome do esgotamento profissional ou estafa profissional, surge pela cronificação de um processo de estresse. Ela é resultado de pressões emocionais repetitivas presentes no ambiente de trabalho. Está associada a ocupações assistenciais, as quais têm contato direto com usuários do serviço, como profissionais da educação e da saúde ${ }^{6}$. Isso ocorre nesses profissionais, dentre outros motivos, devido à divergência entre a expectativa do profissional e a realidade que este encontra no trabalho ${ }^{5}$.

A Síndrome de Burnout caracteriza-se pelos seguintes fatores multidimensionais: Exaustão Emocional, que é a sensação de esgotamento físico e mental; Despersonalização, que está relacionada a alterações de personalidade do indivíduo, com indiferença em relação à população por ele atendida e Reduzida Realização Profissional, na qual o indivíduo apresenta insatisfação com o trabalho, demonstrando querer abandoná-10 ${ }^{6}$.

No Brasil, a Síndrome de Burnout foi oficialmente adicionada às doenças relacionadas à saúde do trabalhador e diretamente vinculadas à atividade laborativa a partir do Decreto no 3.048, de 6 de maio de $1999^{8}$.

Entender quais fatores interferem na saúde do trabalhador e, consequentemente, na sua qualidade de vida é de grande importância para que se possa criar estratégias capazes de atuar diretamente nessas situações do trabalho que geram sofrimento e agravos à saúde ${ }^{2}$.

Devido a essa síndrome estar associada às atividades laborais e interferir diretamente na atuação dos profissionais com o público que atende, este estudo teve como objetivo avaliar a presença da Síndrome de Burnout entre os ACS das equipes de saúde da família do município de Aracaju (SE). 


\section{Métodos}

A pesquisa caracteriza-se como um estudo transversal e quantitativo. A coleta de dados foi realizada entre os meses de setembro de 2012 a janeiro de 2013 com os ACS das equipes de saúde da família do município de Aracaju (SE). Para realização do estudo foi selecionada uma amostra do total de ACS que trabalham no município de Aracaju. Segundo dados disponibilizados pela Secretaria de Saúde deste município, no período da coleta existiam 894 profissionais atuando.

A análise estatística utilizada para o cálculo amostral foi a Determinação do tamanho da amostra com base na estimativa da proporção populacional. Considerando o intervalo de confiança de $90 \%$ e o erro máximo de estimativa de 5\%, o tamanho da amostra foi de 271 ACS.

Foram incluídos na amostra os ACS que trabalham nas equipes de saúde da família de Aracaju e que aceitaram, voluntariamente, participar da pesquisa e assinaram o Termo de Consentimento Livre e Esclarecido. Foram excluídos da pesquisa, os ACS que não estiveram presentes no momento da coleta ou que se recusaram a participar da mesma.

Para a aplicação do instrumento de pesquisa, foi utilizada a forma tradicional, com distribuição dos questionários pessoalmente e posterior recolhimento dos mesmos. Antes do início da coleta foi apresentada a finalidade do trabalho e o Termo de Consentimento Livre e Esclarecido. Posteriormente, os voluntários responderam a três questionários autoaplicáveis: 1) um questionário previamente elaborado pelos autores, que verificava a situação socioeconômica e ocupacional dos profissionais; 2) a versão reduzida do questionário Job Stress Scale (JSS) para avaliar a exposição ao estresse no trabalho e 3) o questionário Maslach Burnout Inventory (MBI), para identificação de sinais e sintomas relacionados à Síndrome de Burnout.

A versão reduzida do questionário JSS possui 17 itens que avaliam: demanda psicológica no trabalho, grau de controle sobre o trabalho e apoio social neste. O cálculo do escore foi dado, primeiramente, pela soma dos itens de cada dimensão: a dimensão demanda pode variar entre 5 e 20; quanto maior o valor, maior a demanda psicológica que o trabalhador recebe no trabalho. A dimensão controle pode variar entre 6 e 24; quanto maior o valor, maior o controle do profissional sobre o desenvolvimento do trabalho. A dimensão apoio social pode variar entre 6 e 24; quanto maior o valor, melhor apoio social o trabalhador recebe em seu ambiente de trabalho?.

Para diferenciar os graus alto e baixo de cada dimensão foram calculadas as medianas. Posteriormente, as variáveis demanda psicológica e controle sobre o trabalho, e seus respectivos graus (alto e baixo) foram combinados com o propósito de se construir os quadrantes do modelo bidimensional: Alta exigência (combinação de alta demanda e baixo controle); Trabalho ativo (combinação de alta demanda e alto controle); Baixa exigência (combinação de baixa demanda e alto controle) e Trabalho passivo (combinação de baixa demanda e baixo controle). Os profissionais que apresentaram alta exigência no trabalho possuem maior exposição ao estresse ocupacional; os que estão no grupo de trabalho ativo e trabalho passivo apresentam exposição intermediária ao estresse ocupacional e aqueles que têm baixa exigência no trabalho classificam-se como não expostos ao estresse ocupacional. A dimensão apoio social foi avaliada de forma isolada ${ }^{10}$.

O MBI possui 22 afirmações sobre sentimentos e atitudes relacionados ao trabalho. Divide-se nos três aspectos relacionados à síndrome: exaustão emocional, despersonalização e o envolvimento pessoal no trabalho. Os escores totais foram calculados para cada uma das três dimensões e pontos de corte foram utilizados para classificar em níveis alto, médio e baixo. Para exaustão emocional, a pontuação maior ou igual a 27 indica alto nível; de 19 a 26, nível moderado; e menor que 19, nível baixo. Para despersonalização, pontuações iguais ou maiores que 10 indicam alto nível; de seis a nove, nível moderado; e menores de seis, nível baixo. Na pontuação relacionada ao envolvimento pessoal, pontuações de zero a 33 indicam alto nível; de 34 a 39, nível moderado; e maior ou igual a 40, baixo. A combinação dos níveis encontrados de exaustão, despersonalização e envolvimento pessoal define o grau de esgotamento ${ }^{4,10}$.

Além da relação entre a Síndrome de Burnout e a exposição do risco ao estresse, foram avaliadas a correlação de variáveis sociodemográficas dos ACS (idade, escolaridade e renda familiar) e laborais (tempo de trabalho, presença de cortiços e favelas na microárea e se trabalha em outro local) com a doença, pois essas características, também, podem favorecer o aparecimento da mesma.

$\mathrm{O}$ projeto de pesquisa foi submetido ao Comitê de Ética e Pesquisa da Universidade Tiradentes. Todos os ACS que concordaram em participar da pesquisa assinaram um Termo de Consentimento Livre e Esclarecido, conforme 
Resolução 196/96 de 10 de outubro de 1996, do Conselho Nacional de Ética em Pesquisa ${ }^{11}$.

\section{Resultados}

Dentre os 271 questionários da amostra, 14 não foram respondidos e 35 foram excluídos devido ao mau preenchimento dos JSS e MBI. Ao final da pesquisa foram utilizados 222 questionários, equivalente a $81,9 \%$ da amostra inicial.

A maioria dos ACS pesquisados era do sexo feminino $(87,8 \%)$, na faixa etária entre 30 a 49 anos $(68,9 \%)$, de raça/cor parda (64\%) e católico $(57,6 \%)$ (Tabela 1$)$. Em relação à escolaridade, $58,5 \%$ possuem $2^{\circ}$ grau completo. Dentre os profissionais pesquisados, $48,2 \%$ são casados ou tem união estável, $68,5 \%$ vivem com 1 a 2 salários mínimos, 56,3\% tem filhos, e destes, $60,9 \%$ tem 1 ou 2 (Tabela 2).

Todos os ACS pesquisados são concursados e têm uma carga horária semanal de 40h. Quando questionados se moram na área onde trabalham, $88,7 \%$ afirmaram que sim. $49,5 \%$ dos ACS traba-

Tabela 1. Distribuição dos Agentes Comunitários de Saúde segundo características sociodemográficas, Aracaju, SE, 2013.

\begin{tabular}{lrr}
\hline Variáveis sociodemográficas & n & $\%$ \\
\hline Sexo & & \\
Feminino & 195 & 87,8 \\
Masculino & 27 & 12,2 \\
Idade (anos) & & \\
Não informado & 16 & 7,2 \\
Até 29 & 45 & 20,3 \\
De 30 a 39 & 106 & 47,7 \\
De 40 a 49 & 47 & 21,2 \\
Acima de 50 & 8 & 3,6 \\
Raça/Cor & & \\
Não Informado & 5 & 2,3 \\
Indígena & 3 & 1,4 \\
Parda & 142 & 64 \\
Preta & 42 & 18,9 \\
Branca & 23 & 10,4 \\
Amarela & 4 & 1,8 \\
Outras & 3 & 1,4 \\
Religião & & \\
Não informado & 2 & 0,9 \\
Católica & 128 & 57,7 \\
Evangélica & 45 & 20,3 \\
Testemunha de Jeová & 6 & 2,7 \\
Espírita & 16 & 7,2 \\
Protestante & 1 & 0,5 \\
Outras & 24 & 10,8 \\
\hline & & \\
\hline & & \\
& &
\end{tabular}

lham há mais de 10 anos nessa profissão e 98,2\% já realizaram capacitação específica para atuar como ACS. Dentre os entrevistados, $67,6 \%$ não possuem em sua microárea cortiços, favelas e/ou invasões. $12,2 \%$ necessitaram faltar ao trabalho, principalmente, por problemas relacionados à saúde e $11,3 \%$ afirmaram trabalhar em outro local (Tabela 3).

Em relação ao questionário JSS, que avalia a exposição ao estresse no trabalho, foi observado que $72,5 \%$ dos ACS apresentam alta demanda psicológica e $81,5 \%$ alto controle sobre o trabalho. Ao realizar o cruzamento desses fatores, foi observado que $57,2 \%$ dos profissionais apresentam alta demanda psicológica associada a alto controle sobre o trabalho, que segundo o questionário, equivale a trabalho ativo, apresentando exposição intermediária ao estresse ocupacional (Figura 1).

O JSS avalia ainda o grau de apoio social recebido pelos ACS, com perguntas relacionadas ao ambiente de trabalho e ao relacionamento com os colegas. Nessa pesquisa foi observado que $75,6 \%$ possuem um alto apoio social.

Tabela 2. Distribuição dos Agentes Comunitários de Saúde segundo características sociodemográficas, Aracaju, SE, 2013.

\begin{tabular}{lrr}
\hline \multicolumn{1}{c}{ Variáveis sociodemográficas } & $\mathbf{n}$ & \multicolumn{1}{c}{$\%$} \\
\hline Escolaridade & & \\
$\quad$ Não informado & 4 & 1,8 \\
1 $^{\circ}$ grau completo & 1 & 0,5 \\
$2^{\circ}$ grau incompleto & 15 & 6,8 \\
$2^{\circ}$ grau completo & 130 & 58,6 \\
Universitário incompleto & 30 & 13,5 \\
Universitário completo & 31 & 14 \\
Pós-graduado & 11 & 5 \\
Estado Civil & & \\
Não informado & 6 & 2,7 \\
Solteiro (a) & 86 & 38,7 \\
Divorciado (a) / Separado (a) & 19 & 8,6 \\
Casado (a) & 82 & 36,9 \\
União Estável & 25 & 11,3 \\
Viúvo (a) & 4 & 1,8 \\
Renda familiar (salários mínimos) & & \\
Não informado & 6 & 1,8 \\
De 1 a 2 & 152 & 68,5 \\
De 2 a 3 & 35 & 15,8 \\
De 3 a 5 & 22 & 9,9 \\
Mais de 5 & 9 & 4,1 \\
Filhos & & \\
Não informado & 6 & 16,7 \\
Sim & 125 & 56,3 \\
Não & 60 & 27 \\
\hline
\end{tabular}


O outro questionário analisado foi o MBI, que avalia a presença da Síndrome de Burnout em ACS. Dos profissionais estudados, 57,7\%

Tabela 3. Distribuição dos Agentes Comunitários de Saúde segundo características laborais, Aracaju, SE, 2012.

\begin{tabular}{lrr}
\hline \multicolumn{1}{c}{ Variáveis laborais } & n & $\%$ \\
\hline Mora na área onde trabalha & & \\
Não informado & 3 & 1,4 \\
Sim & 197 & 88,7 \\
Não & 22 & 9,9 \\
Tempo de trabalho (anos) & & \\
Não informado & 5 & 2,3 \\
Até 01 & 3 & 1,4 \\
De 01 a 05 & 55 & 24,8 \\
De 05 a 10 & 49 & 22,1 \\
Acima de 10 & 110 & 49,5 \\
Capacitação & & \\
Não informado & 2 & 0,9 \\
Sim & 218 & 98,2 \\
Não & 2 & 0,9 \\
Cortiços, favelas e/ou invasões na microárea & \\
Não informado & 4 & 1,8 \\
Sim & 68 & 30,6 \\
Não & 150 & 67,6 \\
Faltou ao trabalho no último mês & & \\
Não informado & 2 & 0,9 \\
Sim & 27 & 12,2 \\
Não & 193 & 86,9 \\
Trabalha em outro local & & \\
Não informado & 3 & 1,3 \\
Sim & 25 & 11,3 \\
Não & 194 & 87,4 \\
\hline & &
\end{tabular}

Figura 1. Agentes Comunitários de Saúde segundo as categorias de estresse no trabalho. apresentam grau moderado ou grave de exaustão emocional; $51,8 \%$ apresentam grau moderado ou grave de despersonalização e $59 \%$ de moderado a alto envolvimento pessoal no trabalho. Em relação à Síndrome de Burnout, 59,9\% não apresentaram tendência à doença; $10,8 \%$ apresentaram moderada tendência à doença e 29,3\% apresentarem características equivalentes à Síndrome de Burnout.

Quando realizadas as correlações entre a Síndrome de Burnout e as variáveis sociodemográficas não foram observadas diferenças significativas em relação à idade $(\mathrm{p}<0,6151)$, escolaridade ( $\mathrm{p}<0,7816)$ e renda familiar $(\mathrm{p}<0,7612)$. Assim como nas seguintes variáveis laborais não houve diferenças significativas: tempo de trabalho $(\mathrm{p}<$ $0,5187)$, presença de cortiços e favelas na microárea $(\mathrm{p}<0,7474)$ e se trabalha em outro local ( $\mathrm{p}$ $<0,0570)$.

Quando avaliadas as dimensões do questionário JSS com a Síndrome de Burnout, observouse que não existe correlação significativa com nenhuma delas: demanda $(\mathrm{p}<0,8744)$, controle ( $\mathrm{p}$ $<0,4979)$ e apoio social $(0,4658)$. Apesar do percentual significativo do Trabalho Ativo $(57,2 \%)$, também não houve diferença significativa entre este e a doença.

\section{Discussão}

Esta pesquisa possibilitou identificar a presença da Síndrome de Burnout entre os ACS do município de Aracaju e quais fatores estressantes podem estar influenciando no aparecimento da mesma.

Dentre as variáveis sociodemográficas é importante ressaltar a frequência elevada do sexo feminino. A presença de mulheres como ACS é marcante desde o início do programa. Maria Fátima de Sousa, que participou da construção do PACS, afirma que as mulheres "associaram-se sem pestanejar ao PACS como um projeto pedagógico de nação"12.

Apesar de não haver correlação significativa nesse estudo entre a idade e a presença da Síndrome de Burnout, este é um ponto a se destacar por não corroborar com outros estudos realizados com profissionais da Atenção Primária. Os estudos realizados por Silva e Menezes ${ }^{4}$ com ACS de uma região do município de São Paulo demonstraram que existe um maior risco de despersonalização em pessoas mais jovens. Ratificando esse estudo, uma pesquisa realizada por Trindade et al. ${ }^{13}$ com trabalhadores das equipes de saúde da 
família apresentou significância estatística entre as subescalas do Inventário de Burnout e a idade, onde os mais jovens obtiveram escores superiores nas subescalas de exaustão emocional e despersonalização. Isso pode ser associado à inexperiência presente nos trabalhadores que iniciam a carreira e a frustração ao perceber que aquela profissão não atinge os seus anseios.

Outra questão que não apresentou correlação significativa nesta pesquisa, mas de relevância para discussão diz respeito ao tempo de trabalho. No estudo realizado por Trindade et al. ${ }^{13}$, trabalhadores não esgotados afirmaram, através de relatos, que o tempo de experiência é determinante para o enfrentamento de situações problemáticas em seu trabalho, pois facilita manter a calma, racionalizar diante de situações estressantes e diminuir a angústia durante a resolução dos problemas. Além disso, o longo tempo de trabalho reflete uma baixa rotatividade dos trabalhadores, possibilitando ao ACS uma maior vinculação com a comunidade e o entendimento de seu papel dentro da equipe ${ }^{2}$.

Em relação à existência de outro trabalho paralelo e à presença da Síndrome de Burnout, também não se observou diferença significativa entre as frequências. Um trabalho realizado por Albuquerque et al. ${ }^{14}$, que discute essa questão, observou que a Síndrome de Burnout aparece com menos frequência $(29,55 \%)$ entre profissionais que têm outro emprego. $\mathrm{O}$ autor justifica que a ausência da doença, apesar de maior sobrecarga de trabalho, pode ocorrer pela satisfação com a renda mensal. Além disso, o outro emprego pode proporcionar menos tensão emocional, diminuindo, assim, o comprometimento psicológico. Corroborando com esse estudo, Braga et al. ${ }^{15}$ observou que profissionais de nível superior apresentam menor número de transtornos mentais comuns, estando associado à possibilidade de ter outros vínculos de trabalho e maior remuneração.

As entrevistas realizadas por Trindade et al. ${ }^{13}$ identificaram que entre os sujeitos esgotados existem maiores problemas de relacionamento interpessoal. Em sua pesquisa, a falta de apoio na equipe de trabalho provocou maiores insatisfação e desgaste no trabalho. Shimizu e Carvalho Júnior ${ }^{16}$ relatam, a partir de pesquisa realizada com trabalhadores da atenção primária, que dificuldades na comunicação entre chefia e subordinados, falta de integração no ambiente de trabalho e falta de apoio das chefias para o desenvolvimento profissional interferem negativamente no processo de trabalho em equipe e dificultam a cogestão do trabalho dentro da Equipe de Saúde da Família. Apesar do trabalho em questão não apresentar correlação significativa entre a Síndrome de Burnout e a dimensão apoio social, este é um ponto importante a analisar. Pereira ${ }^{6}$ cita a falta de comunicação no trabalho - onde os funcionários não são consultados ou informados de alterações no trabalho e quais objetivos este pretende alcançar - como um fator importante para o aparecimento da Síndrome de Burnout.

Dentre as dimensões do MBI está a despersonalização, que se caracteriza pelo distanciamento emocional em relação aos colegas de trabalho e às pessoas a quem o profissional deve prestar assistência. Neste estudo, essa foi a dimensão que apareceu com menos frequência, corroborando com as pesquisas realizadas por Silva e Menezes ${ }^{4}$ e Telles e Pimenta ${ }^{7}$ com ACS.

A dimensão que merece mais destaque nesse estudo é a Exaustão Emocional, sendo a que necessita ser trabalhada prioritariamente. A investigação realizada por Telles e Pimenta ${ }^{7}$ observou que ACS emocionalmente esgotados ocasionam um comprometimento da competência para realização de sua função e perda de sua autoestima. Esse desgaste pode se justificar pela tensão emocional gerada pelo contato diário com as pessoas que atendem.

O estudo realizado por Silva e Menezes ${ }^{4}$ observou que $24,1 \%$ dos ACS apresentaram a Síndrome de Burnout. Outra pesquisa realizada por Albuquerque et al. ${ }^{14}$ com profissionais da Estratégia de Saúde da Família de João Pessoa (PB) afirmou que $42,13 \%$ destes profissionais apresentam a síndrome já desenvolvida ou em desenvolvimento. Nesta pesquisa, também foi observado que no município de Aracaju existe um percentual de $29,3 \%$ desses profissionais com a Síndrome de Burnout. Isso pode ser justificado pelo grande envolvimento dos ACS com sua comunidade e a frustação relacionada à ineficácia da resolutividade de seus problemas, que depende do envolvimento de toda equipe multiprofissional.

Sabe-se que a presença de doenças ocupacionais, além de prejudicar a saúde dos trabalhadores, interfere diretamente no desempenho das atividades dos trabalhadores e, consequentemente, na saúde da comunidade por ele assistida. Diante disso, é de grande importância que as instituições conheçam a Síndrome de Burnout, avaliem quais fatores podem contribuir para o aparecimento da mesma e de que forma interfere na saúde de seus profissionais, para que se possam criar estratégias de intervenção, buscando a prevenção de problemas psicossociais em seus trabalhadores ${ }^{6}$. 
É relevante criar espaços coletivos dentro da instituição para o compartilhamento de sentimentos. Resende et al. ${ }^{1}$ afirmam a importância da criação de espaços de reflexão e escuta sobre a definição do papel dos ACS, através de programas institucionais específicos para esses profissionais. Complementando essa proposta, Telles e Pimenta $^{7}$ sugerem intervenções psicológicas, com objetivo de fortalecer a rede de suporte social e amenizar os efeitos das situações estressantes.

Outra questão a se destacar é a maior valorização que deve ser dada a esses trabalhadores pelas chefias e pela equipe multiprofissional, enfatizando as qualidades de cada profissional e a importância de seu papel na equipe de saúde da família. A valorização das atividades dos ACS pelos outros profissionais possibilita um melhor desenvolvimento e bem-estar no ambiente de trabalho².

\section{Considerações finais}

O espaço de trabalho, muito além de necessário para subsistência, deveria ser um local de prazer. O trabalho está diretamente relacionado à identidade individual, no entanto, devido a diversas questões relacionadas ao trabalho nem sempre este proporciona satisfação pessoal, provocando desinteresse, irritação e exaustão, interferindo diretamente na qualidade do serviço ${ }^{6}$. Neste estudo, observou-se que um número elevado de ACS do município de Aracaju apresenta a Síndrome de Burnout em desenvolvimento ou características compatíveis com a mesma.

A Estratégia de Saúde da Família é um dos pilares para a consolidação do SUS, havendo a necessidade de sensibilização dos gestores acerca de investimentos permanentes para implantação das equipes e qualificação de seus trabalhadores. Associado a isso, devem ser criadas medidas que controlem os níveis de estresses dos profissionais da atenção primária, através da detecção precoce de fatores estressores e da busca de estratégias coletivas para enfrentamento desse quadro, favorecendo assim a qualidade de vida dos mesmos e, consequentemente, a assistência prestada à população por eles atendida ${ }^{13}$.

É importante a realização de novos estudos com esses profissionais, principalmente qualitativos, para que se possa conhecer mais especificamente os principais fatores que possibilitam o aparecimento da Síndrome de Burnout.

\section{Colaboradores}

CM Mota trabalhou no delineamento do estudo, coleta, análise e interpretação dos dados, na redação do artigo e revisão da versão final. PS Nunes e GS Dosea realizaram a orientação da pesquisa e da elaboração do artigo. 


\section{Referências}

1. Resende MC, Azevedo EGS, Lourenço LR, Faria LS, Alves NF, Farina NP, Silva NCO, Oliveira SL. Saúde mental e ansiedade em agentes comunitários que atuam em saúde da família em Uberlândia (MG, Brasil). Cien Saude Colet 2011; 16(4):2115-2122.

2. Santos IER. Estresse ocupacional em agentes comunitários de saúde da cidade de Aracaju-Sergipe [dissertação]. Aracaju: Universidade Tiradentes; 2011.

3. Brasil. Ministério da Saúde. Portaria n. ${ }^{\circ} 2.488$, de 21 de outubro de 2011. Aprova a Política Nacional de Atenção Básica, estabelecendo a revisão de diretrizes e normas para a organização da Atenção Básica, para a Estratégia de Saúde da Família (ESF) e o Programa de Agentes Comunitários de Saúde (PACS). Diário Oficial da União 2011; 21 out.

4. Silva ATC, Menezes PR. Esgotamento profissional e transtornos mentais comuns em agentes comunitários de saúde. Rev Saude Publica 2008; 42(5):921-929.

5. Maia LDG, Silva ND, Mendes PHC. Síndrome de Burnout em agentes comunitários de saúde: aspectos de sua formação e prática. Rev Brasileira de Saúde ocupacional 2011; 36(123):93-102.

6. Pereira AMTB. Burnout: Quando o trabalho ameaça o bem-estar do trabalhador. São Paulo: Casa do psicólogo; 2008.

7. Telles SH, Pimenta AMC. Síndrome de Burnout em Agentes Comunitários de Saúde e Estratégias de Enfrentamento. Saúde Soc 2009; 18(3):467-478.

8. Brasil. Decreto n. ${ }^{\circ} 3.048$, de 6 de maio de 1999. Aprova o Regulamento da Previdência Social, e dá outras providências. Diário Oficial da União 1999; 7 maio.

9. Costa MAS. O estresse no trabalho e auto-avaliação da saúde entre os trabalhadores da enfermagem das unidades de urgências e emergências da Secretaria Municipal de Saúde de Campo Grande/MS, 2010 [dissertação]. Rio de Janeiro: Escola Nacional de Saúde Pública Sérgio Arouca; 2010
10. Santos PG. O Estresse e a Sindrome de Burnout em enfermeiros bombeiros atuantes em Unidades de Pronto-Atendimento (UPAS) [dissertação]. Rio de Janeiro: Universidade Federal do Estado do Rio de Janeiro; 2010.

11. Brasil. Normas para pesquisa envolvendo seres humanos (Res. CNS n.o 196/96 e outras). Brasília: Editora Ministério da Saúde; 2003.

12. Sousa MF. Agentes Comunitários de Saúde: choque de povo. São Paulo: Hucitec; 2001.

13. Trindade LL, Lautert L, Beck CLC, Amestoy SC, Pires DEP. Estresse e síndrome de burnout entre trabalhadores da equipe de Saúde da Família. Acta Paul Enferm 2010; 23(5):684-689.

14. Albuquerque FJB, Melo CF, Neto JLA. Avaliação da Síndrome de Burnout em Profissionais da Estratégia Saúde da Família da Capital Paraibana. Psicol Refl Crít 2012; 25(3):542-549.

15. Braga LC, Carvalho LR, Binder MCP. Condições de trabalho e transtornos mentais comuns em trabalhadores da rede básica de saúde de Botucatu (SP). Cien Saude Colet 2010; 15(Supl. 1):1585-1596.

16. Shimizu HE, Carvalho Júnior DA. O processo de trabalho na Estratégia Saúde da Família e suas repercussões no processo saúde-doença. Cien Saude Colet 2012; 17(9):2405-2414.

Artigo apresentado em 15/03/2013

Aprovado em 27/03/2013

Versão final apresentada em 28/03/2013 